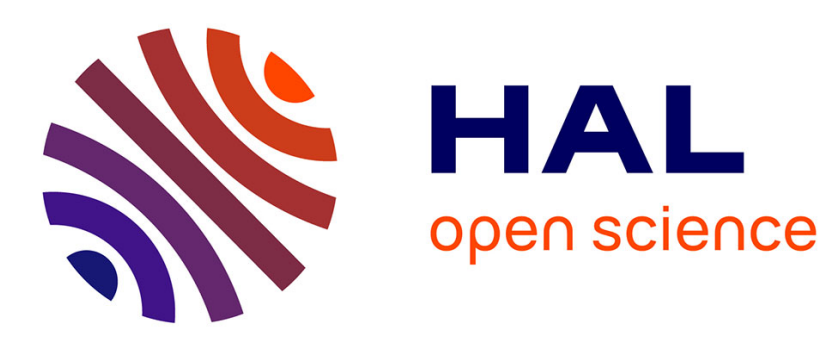

\title{
De l'Égypte à l'Éthiopie, quand la puissance se déplace en Afrique nilotique
}

Pierre Blanc

\section{To cite this version:}

Pierre Blanc. De l'Égypte à l'Éthiopie, quand la puissance se déplace en Afrique nilotique. Confluences Méditerranée, 2014, Afriqu'Orient, 90, pp.123-139. 10.3917/come.090.0123 . halshs-01109437

\section{HAL Id: halshs-01109437 \\ https://shs.hal.science/halshs-01109437}

Submitted on 6 Feb 2020

HAL is a multi-disciplinary open access archive for the deposit and dissemination of scientific research documents, whether they are published or not. The documents may come from teaching and research institutions in France or abroad, or from public or private research centers.
L'archive ouverte pluridisciplinaire HAL, est destinée au dépôt et à la diffusion de documents scientifiques de niveau recherche, publiés ou non, émanant des établissements d'enseignement et de recherche français ou étrangers, des laboratoires publics ou privés. 


\title{
DE L'EGYPTE À L'ETHIOPIE, QUAND LA PUISSANCE SE DÉPLACE EN AFRIQUE NILOTIQUE
}

\author{
Pierre Blanc
}

L'Harmattan | «Confluences Méditerranée »

2014/3 N 90 | pages 123 à 139

ISSN $1148-2664$

ISBN 9782343045627

Article disponible en ligne à l'adresse :

https://www.cairn.inforevue-confluences-mediterranee-2014-3-page-123.htm

Distribution électronique Cairn.info pour L'Harmattan.

(C) L'Harmattan. Tous droits réservés pour tous pays.

La reproduction ou représentation de cet article, notamment par photocopie, n'est autorisée que dans les limites des conditions générales d'utilisation du site ou, le cas échéant, des conditions générales de la licence souscrite par votre établissement. Toute autre reproduction ou représentation, en tout ou partie, sous quelque forme et de quelque manière que ce soit, est interdite sauf accord préalable et écrit de l'éditeur, en dehors des cas prévus par la législation en vigueur en France. Il est précisé que son stockage dans une base de données est également interdit. 


\section{Pierre Blanc}

enseignant-chercheur, Bordeaux Sciences Agro et Sciences Po Bordeaux (LAM).

\section{De l'Egypte à l'Ethiopie, quand la puissance se déplace en Afrique nilotique}

"L'Egypte est un don du Nil ». Cette formule qui nous vient d'Hérodote a été encore vraie dans la période contemporaine, tellement l'Egypte a fait de ce fleuve l'allié de son développement. Profitant de son avance politique et militaire et de ses atouts géostratégiques, le pouvoir central égyptien a pu valoriser les ressources qu'offre le fleuve en termes de sécurisation alimentaire et énergétique. Mais l'affirmation des Etats en amont du bassin du Nil modifie aujourd'hui le statu quo. Sortie de ses vicissitudes d'un "présent historique "à tout le moins tragique, l'Ethiopie est à l'avant-garde des Etats d'amont qui remettent en cause l'avantage de l'Egypte. En surplomb de la question de l'eau, c'est tout un réaménagement du rapport de force qui se noue dans le bassin du Nil, et c'est donc en ces termes que nous décryptons ici la donne hydropolitique; en sachant que les rapports de puissance n'ont pas forcément le dernier mot dans les relations internationales.

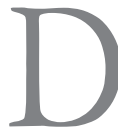

ans un article récent, Kattalin Gabriel-Oyhamuburu s'interroge sur un retour de la géopolitique des ressources ${ }^{1}$. Elle y répond par l'affirmative en précisant que, dans un contexte 
de raréfaction des richesses du sol et du sous-sol, les rivalités d'acteurs s'aiguisent autour de "nouds géostratégiques qui regorgent de ressources énergétiques ou bydriques ». Selon elle, ce sont désormais les deux grands de la planète - les Etats-Unis et la Chine -, ainsi que les puissances émergentes qui sont les plus concernés par cette géopolitique ; une géopolitique qui n'est plus zonale ("vecteur de puissance par le contrôle d'une région ou du monde ») mais objectale ("contrôle des ressources comme seul vecteur de puissance »). Même si cette dichotomie semble quelque peu excessive ${ }^{2}$, il n'en demeure pas moins que la compétition internationale sur les ressources gagne en intensité, notamment sous l'effet de l'accroissement démographique. Cette situation est particulièrement prégnante dans le bassin du Nil où les acteurs impliqués se trouvent dans une recherche in situ de ressources plus que dans une projection vers des territoires lointains comme le font actuellement les grandes puissances : c'est bien l'eau du Nil qui est en jeu pour les Etats riverains et c'est bien la question alimentaire et celle de l'énergie qui sont sous-tendues par cette compétition.

L'acuité nouvelle de la question du Nil tient au fait que les Etats d'amont, sous l'influence éthiopienne, cherchent à s'approprier davantage une ressource qui émane de leur territoire. Plus que jamais, ils usent ainsi de la rhétorique d'une "souveraineté territoriale absolue ", supposée justifier le plein usage de leurs ressources, quand l'Egypte est rivée sur le "principe de première appropriation ", dans un même objectif de légitimation mais fondé sur l'antériorité. En quelque sorte, les droits de la géographie s'opposent ici aux droits de l'histoire. Depuis un siècle, l'Egypte a en effet usé de sa puissance pour pousser plus avant son avantage sur la maitrise de la ressource hydrique. Cependant, depuis le début du $21^{\mathrm{e}}$ siècle les Etats de l'amont du bassin font valoir la leur, ce que les Etats arabes de l'aval ont du mal à admettre, l'Egypte en tête dont la fragilité interne et externe s'impose comme une évidence ${ }^{3}$. Un renversement - ou à tout le moins un rééquilibrage de puissance - est bien en train de se poursuivre dans la région et la question du Nil est très significative de cette réallocation du pouvoir. Mais qu'entend-on par «puissance » ? Pour y répondre, nous retiendrons une définition inspirée du courant réaliste des relations internationales : "On définira la puissance comme une capacité : capacité de faire; capacité de faire faire; capacité d'empêcher de faire; capacité de refuser de faire. " ${ }^{4}$. Même si nous ne pensons pas pouvoir réduire le système de relations internationales en termes de rivalités de puissance, cette définition nous servira de grille pour constater que l'Egypte a eu longtemps la " capacité de 
faire »-accroître sa sécurité alimentaire et énergétique par la maîtrise de l'eau - et «la capacité d'empêcher de faire »- prévenir d'importants prélèvements de « ses » eaux par les pays d'amont du Nil. Cependant, ce temps semble aujourd'hui révolu et, désormais, les pays d'amont ont la "capacité de refuser de faire », autrement dit de contester des accords de partage qui les avaient ignorés. A leur tour, il semble qu'ils aient « la capacité de faire », en particulier l'Ethiopie qui, après bien des décennies de déclassement, s'affirme comme un pays émergent ou en voie d'émergence ${ }^{5}$. Non seulement ce pays s'équipe depuis 2011 d'un barrage de grande ampleur - le barrage de la Renaissance - qui peut affecter le débit du Nil, mais Addis-Abeba a fait montre de diplomatie pour enrôler les Etats de l'amont en vue de rompre une situation imposée par les Etats arabes de l'aval. Si les vecteurs de la puissance sont la démographie, le territoire et le désir d'en être ${ }^{6}$, l'Ethiopie a longtemps manqué du troisième, eu égard aux vicissitudes politiques et militaires qui l'ont affectée. Mais du fait de sa stabilisation et de son volontarisme "développementiste ${ }^{7}$, les relations de puissance s'équilibrent désormais sur le bassin du Nil, d'autant plus que la grande puissance égyptienne est en proie à une transition politique incertaine qui conditionne ses performances économiques et son rayonnement géopolitique. Ainsi, après des décennies de statu quo, un changement est en cours dans la gestion du fleuve.

\section{Le temps de l'hydro-hégémonie égyptienne}

Pays le plus en aval du bassin du Nil, l'Egypte est de loin le plus grand utilisateur des eaux du grand fleuve africain. Si le Nil se trouve depuis longtemps au cœur du rayonnement de l'Egypte, notamment par la pratique de l'agriculture de décrue ${ }^{8}$, c'est essentiellement sous l'autorité de Mohamed Ali qu'il a commencé à faire l'objet d'aménagements hydrauliques d'envergure.

\section{La capacité de faire}

Dans une stratégie d'affirmation de puissance et d'autonomisation vis-à-vis de la tutelle stambouliote, ces travaux devaient faciliter l'alimentation des populations ainsi que le développement des productions d'exportation, en particulier le coton à longues fibres et la 
canne à sucre, et par là, fournir des matières premières à des industries en développement. Sous Mohamed Ali, de grandes canalisations furent ainsi établies, mais le premier barrage construit pour rehausser les eaux du Nil en période d'étiage ne fut cependant envisagé qu'en 1840, avant d'être réalisé entre 1860 et 1880 dans le delta, c'est-à-dire sous le règne du khédive, ou vice-roi, Ismail. Le barrage, qui portait le nom de son grand-père, Mohamed Ali, fut érigé à l'endroit où le Nil se sépare en deux branches, Rosette et Damiette. Il devait relever le niveau des eaux pendant la période d'étiage et, ainsi, faciliter l'irrigation. Ces travaux furent entrepris alors que les prix du coton étaient en pleine envolée, la guerre de Sécession (1860-1866) ayant réduit la présence américaine sur le marché mondial.

Ce rêve de puissance économique et politique, adossé à l'essor de l'irrigation et incarné également par le creusement du canal de Suez (1869), s'acheva avec la fin de la guerre de Sécession et le renversement des cours qui s'ensuivit. La crise des finances publiques provoquée par ce changement de conjoncture fut telle - le surendettement de l'État égyptien provoqua sa mise en faillite en 1876 - que les Britanniques reprirent le financement du canal de Suez au détriment du khédivat qui, jusqu'alors, en partageait la charge avec la France. L'emprise britannique allait durer bien au-delà de l'indépendance et placer l'Égypte, comme à d'autres périodes de son histoire, sous la dépendance d'un centre de pouvoir auquel elle devait fournir de la matière première agricole. C'est d'ailleurs à cette fin que les Britanniques firent établir le premier barrage d'Assouan, inauguré en 1902, pour réguler les crues du Nil et développer l'irrigation.

La construction du barrage dans les années 1960 fut donc une revanche sur l'histoire. "La construction du Haut Barrage, qui apporte le témoignage de la volonté d'une nation et de la détermination d'un peuple, marque notre totale victoire sur l'impérialisme et l'exploitation. Elle constitue un nowvel aspect de notre évolution »" . Ainsi s'exprimait Gamal Abdel Nasser au sujet du barrage, qui fut construit dans les conditions géopolitiques que l'on connait ${ }^{10}$. Avec une population de 20 millions d'habitants croissant à un rythme de $3 \%$ par an, la sécurité alimentaire, que l'on faisait rimer à l'époque avec l'autosuffisance alimentaire, paraissait atteignable en augmentant la productivité agricole. Cet ouvrage était le pendant de la réforme agraire lancée dès 1952 par le nouveau pouvoir égyptien. Ce dessein, né dans les années 1950 et porté dans les années 1960, paraissait d'autant plus souhaitable que l'Égypte se trouvait fortement dépendante pour ses approvisionnements alimentaires, en particulier 
en céréales, qui constituaient - et constituent encore - la base du régime alimentaire national. Dans le contexte de la guerre froide, l'Égypte voulait s'abstraire de l'emprise américaine sur son alimentation. Les États-Unis, dans le cadre du programme PL480 (encore nommé Food for Peace) qui combinait aide alimentaire gratuite et facilités de crédits pour les volumes vendus, en avaient fait un débouché important de leurs excédents et l'Égypte constituait un verrou stratégique, son territoire contrôlant le canal de Suez, crucial pour la maitrise de l'approvisionnement énergétique et le commerce mondial. Le pays souhaitait d'autant plus se défaire de cette dépendance qu'il fut victime de l'arme alimentaire américaine après 1967 - par mesure de rétorsion après son engagement dans la guerre des Six-Jours - au point de devoir mettre en œuvre des cartes de rationnement.

La construction du barrage s'est étalée de 1960 à 1970 et nonobstant nombre de préjudices ${ }^{11}$, elle a permis de réduire la dépendance énergétique de l'Egypte, la centrale hydroélectrique attenante au barrage assurant entre 10 et $20 \%$ de la consommation électrique du pays. Par ailleurs, le barrage a facilité le développement de l'irrigation, qui est la seule manière de faire de l'agriculture dans ce pays très aride. Non seulement la régulation du débit du Nil a permis de doubler, voire tripler les cultures pratiquées sur les mêmes parcelles (extension verticale), mais elle a aussi facilité l'élargissement de la surface irriguée (extension horizontale). Cette extension s'est surtout faite sur les bordures du delta du Nil.

A la fin du $20^{e}$ siècle, l'Egypte a voulu aller plus loin en termes d'extension horizontale en édifiant le canal Zayed supposé permettre l'irrigation d'une partie du Sinaï, tandis qu'il devait approvisionner Israël. Les piètres résultats de ce projet ${ }^{12}$ n'ont pas empêché l'Egypte de relancer le programme dit " de la nouvelle vallée » à partir du barrage d'Assouan. Imaginé dès la période nassérienne et marqué du sceau d'un certain pharaonisme, ce projet visait à désengorger la vallée du Nil par le développement des oasis à l'ouest du fleuve égyptien. Il s'agissait d'élargir la zone de culture dans ces oasis par le seul recours à l'eau souterraine. Mais avec la relance du projet au tournant des années 1990, c'est une canalisation depuis Assouan qui fut imaginée. L'ambition de l'ouvrage était telle qu'il fut nommé « la pyramide en creux de l'an $2000 »$. Mais depuis l'inauguration de la station Moubarak en 2003, l'aménagement de la région de Tochka, qui est le premier compartiment d'irrigation projeté, est très en deçà des espérances alors caressées par le régime. 
En dépit des ces échecs récents sur des projets qui devaient permettre de sortir de la vallée, il faut bien admettre que, depuis la révolution des Officiers libres en 1952, l'Egypte a opéré un déploiement hydraulique qui n'aurait pas pu se produire si elle n'avait pas fait valoir ses droits de façon avantageuse sur les eaux du Nil. L'Egypte s'est comportée au vingtième siècle comme un Etat hydro-hégémonique du fait d'un rapport de force militaire qui a compensé sa position en aval du bassin.

\section{Les aménagements hydrauliques sur le Nil}

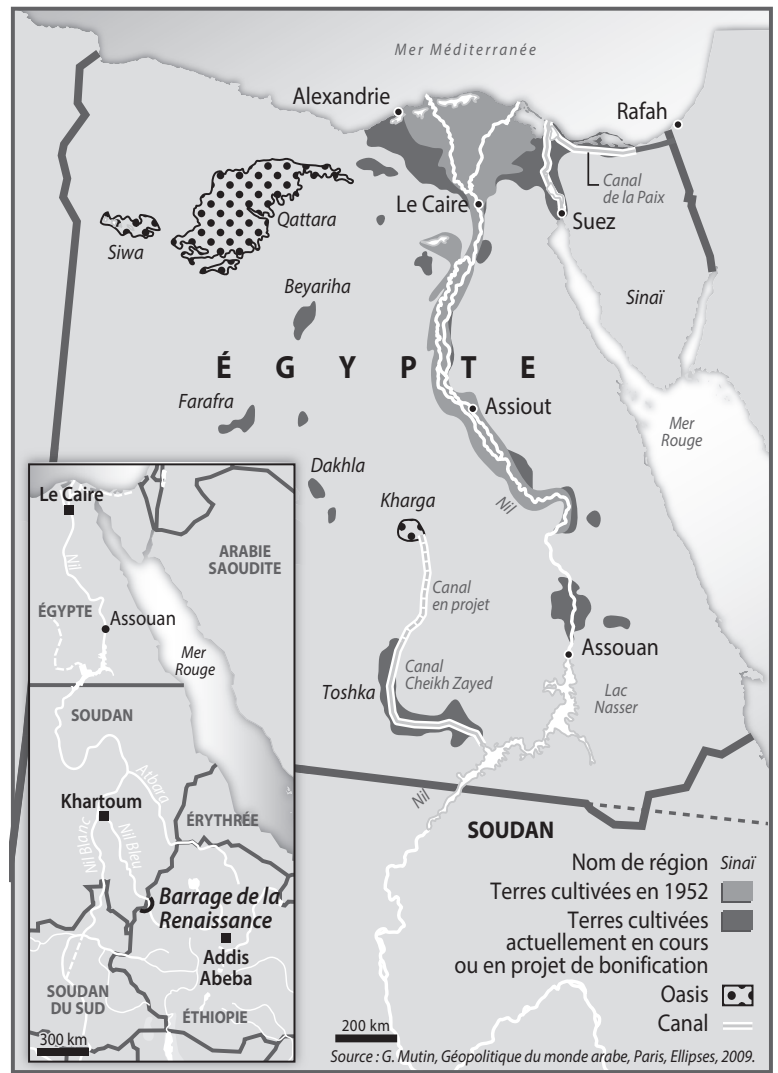

\section{La capacité d'empêcher de faire}

L'affirmation contemporaine de l'Egypte sur le Nil a débuté au lendemain de son indépendance en 1922. Rappelons que l'Angleterre et l'Egypte avaient signé en 1929 un traité par lequel la Couronne 
britannique, puissance coloniale encore installée sur certains territoires du Haut-Nil, avait donné un droit de veto au Caire en cas d'aménagement en amont ${ }^{13}$ et lui avait concédé un droit de prélèvement de 48 milliards de mètres cubes annuels. Seuls 4 milliards de mètres cubes étaient laissés au Soudan et encore, ce pays ne pouvait pas utiliser les eaux en période sèche (de janvier à juillet). Le reste, soit 38 milliards de mètres cubes, n'était pas alloué ou en attente de l'être. Cet accord était donc très avantageux pour l'Egypte, à qui étaient reconnus « des droits naturels et historiques sur les eaux du Nil ». Quant au pouvoir britannique, il était davantage intéressé par le maintien de son contrôle sur le canal de Suez, véritable couloir de passage entre l'océan Indien et la Méditerranée, que par l'usage du Nil.

Après la proclamation d'indépendance du Soudan en 1955 ('indépendance réelle allait intervenir en 1956), Le Caire et Khartoum se lancèrent dans une renégociation de l'accord de 1929. C'est en fait le Soudan qui demanda la révision de cet accord, tant il se considérait lésé (exposition au veto égyptien et faible accès à l'eau). Cet accord marqué du sceau d'un rééquilibrage de la puissance ne fut pas sans tension ${ }^{14}$. Cependant, s'il fut souhaité par le Soudan, l'accord de 1959 ne disconvenait finalement pas à l'Egypte. Engagée alors dans le projet de barrage d'Assouan, elle souhaitait en effet renforcer ses droits en termes de volumes de prélèvements ${ }^{15}$. Ainsi, à l'inverse d'un jeu à somme nulle, le résultat fut avantageux pour les deux Etats signataires. Le droit de veto sur les aménagements en amont, déjà dévolu à l'Egypte, était étendu au Soudan. En excipant de ce droit, les projets futurs de l'Ethiopie, véritable château d'eau du Nil, devenaient désormais improbables. En outre, le Soudan et l'Egypte s'étaient réparti avantageusement des quotas de prélèvements sur le Nil : 75,7 \% du débit annuel ${ }^{16}$ - mesuré à Assouan - pour l'Egypte et 24,3\% pour le Soudan, soit respectivement 55,5 milliards de mètres cubes et 18,5 en année moyenne. L'Egypte gagnait ainsi 7,5 milliards de mètres cubes et le Soudan 14,5. Outre la reconnaissance des droits de ces pays, Khartoum et Le Caire s'autorisaient à construire chacun un barrage : le barrage de Roseires au Soudan et le barrage d'Assouan en Egypte. Pour ce dernier, la submersion des territoires soudanais en amont d'Assouan fut assortie d'un dédommagement égyptien en faveur de Khartoum.

Cet accord, par lequel on passa d'une hydro-hégémonie égyptienne à une hydro-hégémonie partagée entre le Soudan et l'Egypte fut progressivement très mal considéré par les Etats d'amont, dont certains allaient être à leur tour indépendants. Ayant résisté aux tentatives de 
colonisation ${ }^{17}$, l'Ethiopie est le pays qui était le plus concerné par cet accord soudano-égyptien car c'est sur son territoire que se forme l'essentiel du débit du Nil avant de traverser le Soudan puis l'Egypte. Avec le Nil bleu, l'Atbara et une partie du Sobat, les hauteurs éthiopiennes fournissent en effet $86 \%$ du débit du Nil. Le reste provient des autres pays riverains, mais surtout du Soudan. Dans ce pays se forme le Bahr al-Ghazal qui rejoint le Sobat pour former l'essentiel du Nil Blanc dont la confluence avec le Nil Bleu se fait à Khartoum. Avant que le Sobat et le Bahr al-Ghazal se rencontrent au Soudan, les eaux venues des pays d'amont (Rwanda, Tanzanie, Burundi, Kenya) se perdent en partie dans des marécages au sud du Soudan. Ainsi, les autres pays d'amont ne comptent pas beaucoup dans les volumes du cours inférieur du Nil.

La faiblesse du cadre juridique en matière de droits de l'eau et le faible développement des pays d'amont les ont tenus à l'écart de ce partage. Cependant, l'Egypte ayant conscience du caractère contestable de sa position chercha à contenir les critiques des Etats d'amont dans un cadre vaguement coopératif et en élargissant le champ des domaines au-delà de l'eau ${ }^{18}$. De fait, rien n'est venu remettre en question les droits acquis par l'Egypte, ce qui illustre avec force qu'un cadre coopératif peut très bien cacher la confirmation d'une hydro-hégémonie ${ }^{19}$. En parallèle, l'Egypte a même obtenu l'annulation d'un prêt accordé par la Banque africaine de développement pour financer un projet de centrale hydro-électrique sur la partie éthiopienne du Nil Bleu. Mais surtout, elle n'a pas craint de menacer les pays d'amont à plusieurs reprises. En 1978, c'est Anouar el-Sadate lui-même qui utilisa un ton comminatoire contre l'Ethiopie de Mengistu Haile Mariam qui avait envisagé la construction d'un barrage sur le lac Tana. Quant à Boutros BoutrosGhali, bien avant qu'il devienne secrétaire général de l'ONU, il déclara en 1987, en rapport avec un autre projet de barrage sur le lac Tana, que « la prochaine guerre dans la région concernera les eaux du Nil. » L'Egypte craignait d'autant plus le manque d'eau que l'Ethiopie connaissait une sécheresse depuis 1984. Ainsi, en août 1988, le lac Nasser n'avait jamais été aussi vide depuis son remplissage initial ${ }^{20}$. Même si les précipitations reprirent le même mois, peut-on s'étonner dès lors que Le Caire ait eu des plans militaires en direction de ses voisins sudistes - le plan AIDA pour l'Ethiopie et le plan crocodile pour le Soudan - ? Et rien n'indique qu'ils aient disparu depuis. 


\section{La capacité de faire et de refuser de faire : l'affirmation de la puissance éthiopienne}

Aujourd'hui en proie à une mutation interne chaotique, l'Egypte découvre les affres de la contestation hydropolitique. En effet, alors qu'elle fait face à de très fortes urgences politiques et économiques, l'Egypte se voit contestée à l'extérieur, les pays d'amont remettant en question ses « droits historiques » sur le Nil. La montée en puissance de l'Ethiopie, véritable château sur ce grand fleuve africain, constitue un changement majeur dans la région. Autour de ce pays, les Etats d'amont se sont progressivement coalisés pour contester le monopole de l'Egypte et du Soudan sur les eaux du Nil, précisé par les accords de 1959. Cette coalition d'intérêts regroupant d'abord l'Ethiopie, le Kenya, l'Ouganda, le Burundi, le Rwanda et la Tanzanie a débouché le 14 mai 2010 sur la signature d'un accord sur les eaux du Nil. Ce texte qui tend de facto à abroger le traité élaboré en 1929 et amendé en 1959 est l'aboutissement d'une discussion entamée dès 1997 entre pays riverains. Cette année-là, le droit international de l'eau avait franchi un palier certain avec la promulgation, le 21 mai 1997, de la Convention sur le droit relatif aux utilisations des cours d'eau internationaux à des fins autres que la navigation.

Dans le cadre de cette discussion sur une coopération politique sur le bassin du Nil s'inspirant peu ou prou de la convention onusienne de 1997, l'Egypte et le Soudan avaient tenté d'empêcher une remise en question radicale de l'accord de 1959. Le texte déjà quasiment finalisé en 2007 achoppait sur l'article 14 b que seuls les pays d'amont acceptaient en l'état. En effet, cet article semblait, au moins en creux, remettre en question les droits de l'Egypte et du Soudan ${ }^{21}$. Le blocage des discussions né de ces divergences et le besoin de développement de l'Ethiopie expliquent qu'en mai 2010, celle-ci ait promulgué un accord sur le Nil, avec la Tanzanie, le Rwanda, le Kenya et l'Ouganda. En février 2011, ce fut au tour du Burundi de signer cet accord (Cooperative Framework Agreement, CFA). Au lendemain du départ de Hosni Moubarak, ce pays n'était plus soumis aux pressions égyptiennes qui avaient pu se faire sentir au préalable ${ }^{22}$.

Comme cet accord est inspiré de la convention onusienne de 1997, les pays d'amont ont beau jeu depuis sa signature d'insister sur sa légitimité. Cependant, afin de laisser la porte ouverte à une négociation avec les Etats d'aval, il met délibérément en annexe l'article 14 b si 
contesté, en précisant qu'il sera négocié plus tard. De son côté, l'Egypte, en pointe dans le refus de l'accord, prétexte que l'eau du Nil est importante pour elle et le Soudan, bien plus que pour les pays d'amont dont les ressources excèdent les besoins, à la différence des Etats d'aval. Pour prévenir la signature, le gouvernement égyptien a même tenté une " opération séduction ${ }^{23}$ envers les pays d'amont via le financement de projets de développement, tout en usant en parallèle de menaces à peine voilées ou de pressions sur les bailleurs de fonds et la Banque mondiale, appelés à financer les infrastructures hydrauliques de l'Ethiopie.

Il ne fait aucun doute que, parmi les pays d'amont, l'Ethiopie a été de loin la plus active sur le dossier et c'est bien elle qui a agrégé les Etats contestataires. Alors que la période d'instabilité et d'incertitude en Egypte minait sa position d’hydro-hégémonie, cette audace de l'Ethiopie n'était pas fortuite. Ses dirigeants actuels se veulent les hérauts de sa renaissance après trois décennies durant lesquelles ce pays n'a cessé de plonger dans les tragédies les plus sombres. Ce fut en particulier le cas sous le pouvoir hyper-autoritaire de Mengistu (19771991) qui provoqua des famines liées à la collectivisation foncière, aux conflits nés de ces politiques et au déplacement des populations. Minée par les famines et les conflits internes, notamment celui de l'Ogaden (1977-1978), l'Ethiopie fut débarrassée du Négus rouge avec l'arrivée du Tigréen Meles Zenawi en 1991. Cependant, elle perdit l'Erythrée en 1993 et connut avec elle, en 1998, une guerre des frontières qui fit quelque 100000 victimes.

Depuis le début de ce millénaire, Meles Zenawi, décédé en août 2012, a profité de la phase de relative normalisation interne - très relative au demeurant - pour lancer des projets de grande ampleur (routes, voies ferrées) sur le territoire en vue de recouvrer une grandeur passée. Dans le Livre blanc de la stratégie et la sécurité 2002, qui fixe les orientations politiques du pays, il faut souligner la forte occurrence du terme « honte », en référence au sentiment de disqualification né des dérives politiques qui ont conduit ce très ancien phare de l'Afrique loin du concert des nations. Cependant, pour revenir de plain pied dans l'histoire, des urgences s'imposent à l'Ethiopie, qui justifient un volontarisme économique de sa part. Ses besoins de développement sont importants et sa population a dépassé, depuis 2000 ( $c f$. annexe 1), celle de l'Egypte voisine, ce qui obère en particulier sa sécurité alimentaire ${ }^{24}$.

Dans un pays où les fortes irrégularités des précipitations, tant spatiales que temporelles, entravent la capacité de production, les 
attentes en irrigation sont donc massives, aussi bien pour assouvir les besoins nationaux que pour développer les exportations agricoles destinées à financer en partie la modernisation de l'économie. De même, le besoin d'énergie pousse les autorités à promouvoir l'hydroélectricité. Cependant, au-delà de la production énergétique intérieure pour l'industrialisation du pays, l'Ethiopie veut profiter de ses avantages topographiques pour fournir de l'énergie hydro-électrique à son voisinage et y assurer un certain soft power, en l'occurrence un « resources power $»^{25}$, en plus des rentrées financières liées à ces livraisons.

Ainsi, parallèlement à l'accord-cadre de 2010 qui vise une remise en question de l'accord de 1959, le lancement en avril 2011 par l'Ethiopie de la construction du grand barrage du Millénaire, désormais appelé le barrage de la Renaissance éthiopienne - c'est tout dire ! -, figure parmi les plus grands motifs d'inquiétude au Caire. C'est surtout le cas pour la phase de remplissage qui retiendra immanquablement de l'eau dans la partie éthiopienne du Nil Bleu. Ce barrage inquiète plus les Egyptiens que le barrage de Mérowé, construit par le Soudan et dont le remplissage est supposé respecter le quota annuel soudanais. Après qu'il aura été achevé, normalement d'ici 2017, puis rempli selon un délai qui est encore à négocier, 6000 mégawatts devraient être fournis par ce barrage de la Renaissance dont le volume doit atteindre 63 milliards de mètres cubes, ce qui en fera l'un des plus grands barrages du monde et le premier sur le continent africain. Il permettra également de développer l'irrigation sur les zones en aval du barrage ${ }^{26}$, l'amont n'étant pas propice à l'agriculture pour des raisons topographiques. La possibilité d'irriguer de nouveaux périmètres au Soudan et de prévenir les inondations décennales, notamment dans la plaine agricole de la Djezireh et la capitale Khartoum font du Soudan un allié timide du projet éthiopien. Parce que le Soudan se rapproche ainsi de l'Ethiopie, isolant un peu plus l'Egypte sur le plan hydropolitique et parce que ce projet risque de prélever un stock d'eau conséquent, les autorités du Caire sont aujourd'hui très inquiètes. Il faut dire que la sécurité alimentaire $^{27}$ y est déjà menacée ${ }^{28}$ et que les autorités égyptiennes souhaitent élargir l'écoumène en dépassant l'aréisme du pays par la mise en eau de certains secteurs géographiques (Nouvelle vallée, Sinaï, delta du Nil).

Avant d'être expulsé du pouvoir, Mohamed Morsi s'était ainsi montré menaçant, précisant, dans le cadre d'une réunion politique consacrée à la question le 10 juin 2013, que «toutes les options sont sur la table ». Au préalable, il avait semblé plus arrangeant mais tout laisse à 
penser que sa perte de légitimité l'avait poussé à durcir ses positions sur une question de sécurité nationale. Cependant, cette réunion politique, qui aurait dû se tenir dans le secret, fut étonnamment retransmise à l'insu des participants y compris du président lui-même. Le teneur des débats, à contenu très stratégique, et les propos compromettants furent rendus publics avec les effets désastreux qu'on imagine ${ }^{29}$. Sans dire que cette réunion et, plus largement, l'impéritie de Morsi sur le dossier hydropolitique furent la cause de son départ, constatons qu'elles se sont ajoutées à l'ensemble des griefs d'une grande partie de la population contre les Frères musulmans au pouvoir. En tout cas, quelque trois semaines après cette réunion politique, le mouvement Tamarrod parvenait à mobiliser des millions d'Egyptiens, ce qui conduisit l'armée à déposer le président Morsi le 3 juillet 2013.

Avec le nouveau pouvoir du général Al-Sissi, l'heure semble être à la détente sur le dossier. De son côté, l'Ethiopie ne considère pas que son projet de barrage affecte l'intérêt des pays d'aval. Le plan imaginé par l'américain Harold Dewin Hurst dès 1946 ne prévoyait-il pas la construction de barrages en Ethiopie pour optimiser la gestion du Nil à l'échelle du bassin ? Le stockage de l'eau sous des climats moins chauds devait permettre de limiter l'évaporation par rapport à ce qui fut fait par la suite dans la région d'Assouan. Cette rationalité hydrologique fait donc partie du discours actuel en Ethiopie. Il n'empêche : si, à long terme, la construction de barrage ne devrait pas vraiment avoir d'incidences sur le débit en aval - car l'hydroélectricité est préférée à l'irrigation -, la phase de remplissage du barrage de la Renaissance pourrait plus ou moins l'affecter selon sa durée.

\section{La capacité de faire ensemble}

La question du Nil ne concerne pas seulement la discussion d'un nouveau traité et la construction du barrage de la Renaissance. D'autres barrages pourraient être construits, ce qui laisse la porte ouverte à la conflictualité... ou à la coopération. Au-delà de la démographie dont les prévisions sont à peu près prévisibles, deux variables seront déterminantes sur cette question, sans que l'on sache véritablement dans quel sens elles peuvent influer. Une première variable tient aux évolutions climatiques sur le bassin hydrographique du Nil. Cependant, elle demeure très difficile à évaluer tant les prévisions à son sujet se déploient sur un large spectre. Si les différents modèles sont unanimes 
à faire apparaittre une élévation des températures, il n'en est rien du régime de précipitations qui peut augmenter fortement comme diminuer significativement ${ }^{30}$. Evidemment, dans le cas d'un accroissement des précipitations, l'Egypte pourrait se voir rassérénée sur le dossier du Nil, au point même de souhaiter que les pays d'amont aillent plus avant dans la constitution d'un équipement hydraulique supposé diminuer les inondations en aval. A l'inverse, une diminution des précipitations pourrait tendre les relations, même si un stress général peut générer une prise de conscience collective profitable à tous.

Une seconde variable tient à l'évolution des régimes politiques sur le bassin du Nil. En vertu de la «théorie de la paix démocratique » ${ }^{31}$, la démocratisation des régimes pourrait être porteuse de coopération, plus que de tension. Cependant, l'histoire hydraulique montre que, même en présence de régimes autoritaires, la coopération prévaut sur la guerre ${ }^{32}$. Pour l'heure, l'Egypte, qui ne montre pas les signes d'une transition démocratique avérée semble vouloir engager un dialogue, voire une coopération, avec l'Ethiopie. C'est du moins ce que laissent penser les déclarations du nouveau pouvoir égyptien. Mais l'histoire contemporaine nous enseigne qu'il faut demeurer prudent devant ce genre de déclarations qui ont déjà précédé des propos plus comminatoires.

Dans un contexte inédit (importance de la population, équilibre des puissances, incertitudes climatiques), l'Egypte, qui a longtemps eu la main sur le Nil, est le pays qui pourrait avoir le plus à perdre avec la remise en question du statu quo. Cependant, elle dispose encore de marges de manœuvre en matière d'économies d'eau ${ }^{33}$. Ceci renvoie à la responsabilité politique de ce pays qui, dans un contexte d'équilibre de puissance, ne pourra plus faire pression sur ses voisins pour éviter en interne les remises en question de ses politiques hydrauliques. Mais par-delà ses efforts, l'Egypte a tout intérêt, comme les pays du bassin, à rester sur le chemin du dialogue politique qui parait la seule voie porteuse pour tous. La gestion commune et plus équilibrée des eaux du Nil, dont les termes sont encore à définir concrètement, peut permettre la sortie d'un jeu à somme nulle : la sécurité alimentaire et énergétique des uns ne suppose pas que les autres doivent y renoncer. Ainsi, le bassin du Nil qui a vu plusieurs attributs de la puissance se déployer (la capacité de faire, la capacité d'empêcher de faire et la capacité de refuser de faire) gagnerait à coup sûr si la capacité de faire ensemble s'invitait désormais comme un horizon nouveau. L'articulation que l'on observe de plus en plus au nord-ouest de l'Afrique entre Etats arabes et Etats 
subsahariens pourrait donc s'offrir aussi, dans cette partie orientale du continent, comme un dénouement heureux d'une situation régionale où les rapports de puissance sont longtemps restés déséquilibrés.

\section{Annexe 1}

Evolution de la population de l'Egypte et de l'Ethiopie de 1950 à 2050 (source ONU)

\begin{tabular}{|c|c|c|}
\hline & Egypte & Ethiopie \\
\hline Année & \multicolumn{2}{|c|}{ Population en milliers } \\
\hline 1950 & 21514 & 18128 \\
\hline 1955 & 24387 & 19947 \\
\hline 1960 & 27998 & 22151 \\
\hline 1965 & 32084 & 25014 \\
\hline 1970 & 36342 & 28415 \\
\hline 1975 & 40359 & 32570 \\
\hline 1980 & 44932 & 35241 \\
\hline 1985 & 50347 & 40777 \\
\hline 1990 & 56337 & 48043 \\
\hline 1995 & 61168 & 57024 \\
\hline 2000 & 66137 & 66024 \\
\hline 2005 & 71778 & 76167 \\
\hline 2010 & 78076 & 87095 \\
\hline 2015 & 84706 & 98942 \\
\hline 2020 & 91062 & 111521 \\
\hline 2025 & 96989 & 124537 \\
\hline 2030 & 102553 & 137670 \\
\hline 2035 & 107900 & 150731 \\
\hline 2040 & 113001 & 163553 \\
\hline 2045 & 117689 & 175896 \\
\hline 2050 & 121798 & 187573 \\
\hline
\end{tabular}

Notes

1. Kattalin Gabriel-Oyhamuburu, « Le retour d'une géopolitique des ressources ? » L'espace politique, 12, 2010-3.

2. La rivalité au sujet des ressources n'est absolument pas nouvelle et elle a 
De l'Egypte à l'Ethiopie, quand la puissance se déplace en Afrique nilotique

toujours eu partie liée à la géopolitique zonale. Les politiques de projection anglaise, française, allemande puis américaine et soviétique ont toujours combiné la recherche de ressources à la politique d'emprise territoriale.

3. Pierre Blanc, "Egypte : géopolitique d'une fragilité », Confluences Méditerranée, $\mathrm{n}^{\circ} 75$, automne $2010 ; \mathrm{p}$. 13-31.

4. Serge Sur, Relations internationales, éd. Montchrestien, $2000 ;$ p. 229. On pourra également se reporter à l'ouvrage de Pierre Bulher, La puissance au XXIe siècle, CNRS Editions, 2011 (préface d'Hubert Védrine)

5. La Coface qui a inventorié le « top 10 » des pays émergents en 2014 classe l'Ethiopie dans un groupe 2, autrement dit un ensemble de pays dont l'émergence est ralentie par un environnement des affaires très précaire. Lire aussi Laurence Daziano, La nouvelle vague des émergents. Bangla Desh, Ethiopie, Nigeria, Indonésie, Vietnam, Mexique, Fondation pour l'innovation politique (Fondapol), 2013.

6. Pierre Verluise, "Géopolitique. La puissance : Quels sont ses fondamentaux ? », Diploweb, 10 novembre 2013.

7. Au pouvoir depuis 1991, le Front démocratique révolutionnaire du peuple éthiopien (FDRPE) a choisi la voie d'un Developmental State, dont les fondements reposent beaucoup sur le secteur public, mais également, comme le fait remarquer Clélie Nallet, «sur un choix politique de domination du parti et sur un contrôle général de la société ». Clélie Nallet, Quelle success story pour l'Afrique?, Institut de relations internationales et stratégiques (Iris), Afrique décryptage, juillet 2014.

8. Les paysans du Nil pratiquaient la culture de décrue dans des bassins de rétention (hod) construits à cet effet. La submersion durait entre trente et quarante jours; une fois les eaux évacuées, la culture de décrue cédait la place à la culture hivernale, les températures de la vallée n'obligeant pas les plantes au sommeil végétatif. Après le printemps, les sols craquelés par la sécheresse pouvaient recevoir d'autant mieux les eaux des crues chargées en limons, qui constituaient autant de fertilisants naturels, d'où l'appellation parfois utilisée de «culture en limonage ». Bien sûr, la force de la crue déterminait la largeur de la surface irriguée de la vallée.

9. Annabelle Boutet, L'Égypte et le Nil, pour une nouvelle lecture de la question de l'eau, L'Harmattan, 2001 ; p. 194.

10. La question du financement du barrage d'Assouan s'est vite posée. Afin de garder l'Égypte dans leur aire d'influence, les États-Unis et la GrandeBretagne ont accordé au Caire leur soutien technologique et surtout financier par le biais de la Banque internationale pour la reconstruction et le développemen (Bird). Mais, après avoir essayé d'acheter des armes à l'URSS par l'intermédiaire de Tito, l'Égypte essuie en 1955 un refus de financement de la Banque mondiale. Comme ils l'ont fait pour le Liban, où les aménagements sur le Litani risquent de défavoriser l'allié israélien, les Américains usent là aussi de leur influence pour refuser le prêt à l'Égypte tant qu'elle n'annule pas son contrat d'armement. Le président Nasser justifie alors la nationalisation du canal de Suez en 1956, qui doit permettre de percevoir des droits de péage. La France et la Grande-Bretagne, appuyées par Israël, réagissent en octobre 1956 pour reprendre le contrôle du canal, mais les États-Unis, soucieux de ménager les Soviétiques qui viennent d'établir le pacte de Varsovie, parviennent à arrêter l'intervention. Consacré leader du nationalisme arabe, Nasser effectue alors un virage politique net dans la foulée de ces événements. Alors qu'elles étaient plus inspirées par l'idéologie des Frères musulmans, les autorités égyptiennes adoptent 
une orientation socialiste, en particulier en 1958, en acceptant une aide soviétique refusée deux ans plus tôt.

11. Les limons charriés depuis l'Ethiopie voisine sont retenus par le barrage, qui tend ainsi à s'envaser d'année en année. En outre, cette éviction du limon en aval du lac Nasser rend l'agriculture très dépendante de la fertilisation chimique, dont le niveau d'utilisation à l'hectare avoisine celui des pays européens. Ce recours aux engrais expliquerait en partie le développement de certains cancers, quoiqu'il soit difficile de faire la part des choses entre des effluents urbains et industriels non traités, tout aussi toxiques, et les résidus de l'activité agricole. D'autre part, l'eau étant exposée aux fortes températures, sa concentration saline en aval du lac Nasser s'élève, ce qui contribue à la salinisation croissante des sols. Enfin, la quasi-disparition du poisson du Nil, préjudiciable aux paysans égyptiens qui en étaient de gros consommateurs, est à l'évidence liée au barrage autant qu'à des prélèvements massifs. Bien sûr, l'utilisation du lac Nasser comme ressource piscicole rétablit le déficit, mais les paysans ne peuvent pas les pêcher eux-mêmes, avec tout ce que cela comporte pour des budgets fragiles. D'autres effets néfastes sont évoqués régulièrement avec plus ou moins de pertinence : il ne fait aucun doute en effet que la jacinthe d'eau s'est développée du fait de la plus grande stagnation des eaux en aval d'Assouan, ce qui explique par ailleurs la recrudescence de la bilharziose.

12. Aujourd'hui, le canal n'arrive que dans la ville d'Al-Arish en Egypte et l'irrigation du Sinaï est au point mort.

13. Ainsi le paragraphe 4 b stipule-t-il que " aucun ouvrage d'irrigation ou de production d'énergie électrique ne peut être construit, au Soudan ou dans les territoires sous administration britannique, qu'avec le consentement paisible du gouvernement égyptien. »

14. Devant l'intransigeance de l'Egypte en apparence sûre de son fait, le Soudan commença en juillet 1958 à irriguer les terres de la Djezireh à partir du barrage de Managuil situé sur le Nil bleu. Mais alors que les bruits de bottes se faisaient entendre de part et d'autre, une médiation arabe tenta de désamorcer la tension. Le coup d'Etat au Soudan en novembre 1958 facilita la reprise des négociations qui débouchèrent sur l'accord du 8 novembre 1959.

15. Edouard Boinet, Hydropolitique du Nil. Du conflit à la coopération ?, L'Harmattan, 2012.

16. Le débit du Nil à Assouan était de 84 milliards de mètres cubes, auxquels il fallait enlever 10 milliards de mètres cubes liés à la construction de retenues.

17. Hormis la courte parenthèse italienne.

18. On peut mentionner ici les projets Hydromet, Undugu, Tecconile. Le projet Hydromet lancé en 1967 concernait les possibilités d'utilisation du Nil Blanc que l'Egypte n'utilise pas puisque ses eaux se perdent dans les marécages du Sudd (en arabe, "barrière ») au Soudan. L'Undugu ( « fraternité » en swahili) est un projet de coopération économique lancé en 1980. Le Tecconile (Comité intergouvernemental de coopération technique, pour la promotion du développement et la protection de l'environnement) est une instance de coopération technique visant à former les responsables dans le domaine de l'eau, à préserver l'environnement nilotique et à renforcer les capacités institutionnelles dans le secteur de l'eau.

19. Mark Zeitoun et Jeroen Warner, "Hydro-hegemony, a framework for Analysis of Trans-Boundary Water Conflicts, Water Policy, 8(5), 2006 ; p. 435-460. On peut également lire l'article de Ana Cascao, "Changing 
De l'Egypte à l'Ethiopie, quand la puissance se déplace en Afrique nilotique

power relations in the Nile river Basin: Unilateralism vs cooperation? ", Water Alternatives 2(2), 2009 ; p. 245-268.

20. Erlich Haggaï, The Cross and The River, Ethiopia, Egypt and The Nile, Boulder, 2002.

21. Dans l'article $14 \mathrm{~b}$, il est précisé que les Etats conviennent « de ne pas affecter considérablement la sécurité hydrique de tout autre Etat du Bassin du Fleuve du Nil ». Les pays d'aval ont proposé alors que cet article précise "de ne pas affecter défavorablement la sécurité hydrique ainsi que les usages et droits actuels de tout autre Etat du Bassin du Fleuve du Nil » (entendu l'Egypte et le Soudan).

22. Ana Elisa Cascao, "Nile Water Governance », in Seleshi Bekele Awulachew, Vladimir Smakhtin, David Molden et Don Peden, The Nile River Basin, Water, Agriculture, Governance and Livelihoods, Routledge, 2012.

23. Edouard Boinet, op. cit. ; p. 111-113.

24. Alain Gascon, « Oublier Malthus : Ethiopie, la crise alimentaire surmontée ? » Hérodote, La Découverte, 131, 2004/4 ; p. 73-91.

25. Bernice Lee, Felix Preston, Jaakko Kooroshy, Rob Bailey et Glada Lahn, Resources Futures, A Chatham House Report, Londres, décembre 2012.

26. Des études annoncent la possibilité d'irriguer 500000 hectares. «Grand Ethiopian Renaissance Dam project, Benishangul Gumuz, Ethiopia », www. water-technology.net (consulté le 18 juillet 2014).

27. Pierre Blanc et Sébastien Abis, «Egypte, entre insécurités alimentaires et incertitudes géopolitiques », Le Démeter 2015, Economie et stratégies agricoles, septembre $2015 ;$ p. 51-105.

28. Autant pour des raisons sociales et de choix politiques que pour un supposé manque d'eau. Les inégalités sociales conduisent les populations les plus aisées à opérer une transition alimentaire vers plus de produits carnés et donc plus exigeants en calories. D'autre part, signalons le choix des exportations agricoles comme un moyen de financer un endettement progressif, au détriment des produits de base. Sur cette question, on pourra se reporter à l'excellent livre de Timothy Mitchell, Rule of Experts, University of California Press, Berkeley, 2002

29. Un islamiste a soutenu l'idée qu'il fallait aider les rebelles éthiopiens quand un autre participant libéral a insisté pour faire courir la rumeur d'une intensification de la préparation militaire en Egypte.

30. P. Michael Link et al., « Vulnerabilities, Adaptative Capacities and Transboundary Relations in The Nile River Basin in Times of Climates Change ", L'Europe en formation, $n^{\circ} 365 ;$ p. 99-130.

31. Théorie que l'on doit à Mikael Doyle et qui s'inspire de Kant (Vers la paix perpétuelle, publiée en 1775). Elle stipule que deux démocraties ne se font pas la guerre et Doyle, à l'instar de Kant, décrypte les ressorts de cette impossibilité.

32. Aaron Wolf, « Conflict and cooperation along international waterway », Water Policy $n^{\circ} 1$ (1998); p. 251-265.

33. George Mutin, "Quel avenir hydraulique pour l'Egypte ? " in Eau et pouvoir en Méditerranée (dir. Pierre Blanc), Confluences Méditerranée, $n^{\circ}$ 58, été 2006. 